Article

\title{
Association between Dietary Pattern and Incidence of Cholesterolemia in Korean Adults: The Korean Genome and Epidemiology Study
}

\author{
Jieul Lee and Jihye Kim * \\ Department of Medical Nutrition, Graduate School of East-West Medical Science, Kyung Hee University, \\ Yongin 17104, Korea; dlwldmf@naver.com \\ * Correspondence: kjhye@khu.ac.kr; Tel.: +82-31-201-3497
}

Received: 10 August 2017; Accepted: 5 January 2018; Published: 9 January 2018

\begin{abstract}
We examined the gender-specific association between dietary pattern and risk of developing cholesterolemia based on the data from the Korean Genome and Epidemiology study. A total of 7515 individuals aged 40-69 years participated in this study between 2005 and 2010. Dietary intake was assessed by a semi-quantitative food frequency questionnaire. Low HDL cholesterolemia was defined as a plasma HDL-C level $<1.04 \mathrm{mmol} / \mathrm{L}$ (men) or $<1.30 \mathrm{mmol} / \mathrm{L}$ (women), and high LDL cholesterolemia was defined as a plasma LDL-C level $>3.37 \mathrm{mmol} / \mathrm{L}$. Multivariate Cox proportional hazard models were used to examine the risk for incident cholesterolemia according to dietary pattern score. Four dietary patterns were derived by gender using factor analysis: prudent pattern; coffee, fat, and sweet pattern; whole grain (men) or white rice and noodle (women) pattern; and westernized pattern. A prudent pattern was inversely associated with risk of low HDL cholesterolemia in both men (Hazard ratio $(\mathrm{HR})=0.76, p$ for trend $=0.0098)$ and women $(\mathrm{HR}=0.78, p$ for trend $=0.0324)$, whereas the coffee, fat, and sweet pattern was positively associated with risk of high LDL cholesterolemia in men only $(\mathrm{HR}=1.26, p$ for trend $=0.0254)$ after adjustment for potential confounders. Specific dietary patterns were associated with risk of developing cholesterolemia suggesting gender differences.
\end{abstract}

Keywords: dietary pattern; Korean Genome and Epidemiology study; low HDL cholesterolemia; high LDL cholesterolemia; prudent pattern; coffee; fat; sweet pattern

\section{Introduction}

Dyslipidemia is well recognized as a major cause of cardiovascular disease (CVD) [1,2]. According to the U.S. National Health and Nutrition Examination Survey, the prevalence of dyslipidemia was 53\% in U.S. adults [3]. Dyslipidemia is steadily rising in Korea as well as western populations. Data from the Korea National Health and Nutrition Survey reported that the prevalence of dyslipidemia was $57.6 \%$ in men and $60.4 \%$ in women in 2010 [4].

Dietary pattern is an important determinant of chronic diseases such as type 2 diabetes, hypertension, and metabolic syndrome [5-7]. Previous studies have shown the association between dietary pattern and dyslipidemia [7-10]. A Mediterranean diet characterized by high intake of vegetables, fruits, whole grains, fish, legumes, nuts, and olive oil was positively associated with high-density lipoprotein cholesterol (HDL-C) in a Spanish population aged 40-55 years [8]. In a randomized controlled trial, the Dietary Approaches to Stop Hypertension (DASH) diet, which is high in whole grains, vegetables, fruits, legumes, and low-fat dairy products, reduced low density lipoprotein cholesterol (LDL-C), and total cholesterol compared with a control diet in healthy adults aged $>21$ years [11]. A study of Northern Chinese adults aged 20-74 years showed that a snack pattern with high intake of biscuits, fried chips, liquid beverages, sweets, and ice cream was positively associated with serum total cholesterol, LDL-C, and triglyceride (TG) [10]. 
However, the effect of dietary pattern on dyslipidemia has not been confirmed from prospective studies. Furthermore, evidence of the association between dietary pattern and dyslipidemia is very limited for Asian populations.

Dietary patterns vary depending on age, gender, culture, and ethnicity [12]. Koreans, especially older people, consume a very high carbohydrate diet ( $>70 \%$ of energy intake), and such a high carbohydrate intake is associated with changes in blood cholesterol levels among Koreans [13].

In the current study, we investigated the gender-specific association between dietary pattern and risk of developing cholesterolemia (low HDL cholesterolemia or high LDL cholesterolemia) in middle-aged or older Korean adults using data from the Korean Genome and Epidemiology Study (KoGES), which is a large community-based cohort study.

\section{Materials and Methods}

\subsection{Study Population}

This study was based on the KoGES, a large community-based cohort study [14]. The study was conducted to assess the effects of dietary, environmental, and lifestyle determinants on the incidence of chronic diseases in the general Korean population aged 40-69 years living in the Ansan or Ansung areas. Baseline data was collected between 2005 and 2006, and follow-up examinations were performed every 2 years over a 6-year period (2007-2008, 2009-2010). For calculation of follow-up period of participants, data on year, month, and day of follow-up examination were collected. Study participants completed interviewer-administered questionnaires on demographic information and lifestyle factors including dietary habits, their health condition, and medical history, and also anthropometric and biochemical measurements were all conducted biennially.

Among the 7515 subjects who participated at baseline, the following were excluded: 691 participants who refused to participate in the follow-up examination; 3872 participants who had low HDL cholesterolemia at baseline (The participants were excluded to measure incidence of HDL cholesterolemia to predict the risk of low HDL cholesterolemia according to dietary pattern score, which are presented as hazard ratio); 30 participants who had cancer or CVD; 19 participants who had an implausible energy intake ( $<500 \mathrm{kcal}$ or $>6000 \mathrm{kcal}) ; 217$ participants who did not complete a food frequency questionnaire (FFQ); and 31 participants who had missing information. Finally, a total of 2655 subjects (1716 men and 939 women) were entered into the low HDL cholesterolemia analysis. Likewise, among the 7515 subjects who participated at baseline, the following were excluded: 691 participants who refused to participate in the follow-up examination; 2395 participants who had high LDL cholesterolemia at baseline (the participants were excluded to measure incidence of LDL cholesterolemia to predict the risk of high LDL cholesterolemia according to dietary pattern score, which are presented as hazard ratio); 69 participants who had cancer or CVD; 26 participants who had an implausible energy intake ( $<500 \mathrm{kcal}$ or $>6000 \mathrm{kcal}) ; 262$ participants who did not complete a FFQ; and 67 participants who had missing information. Thus, a total of 4005 subjects (2057 men and 1948 women) were entered into the high LDL cholesterolemia analysis.

The study protocol was approved by the Institutional Review Boards of the Korea Centers for Disease Control and Prevention and Kyung Hee University (KHSIRB-16-022). Written informed consent was obtained from all participants.

\subsection{Dietary Assessment}

Dietary intake was assessed by a validated 106-item, semi-quantitative FFQ [15] at baseline. All study procedures were conducted by trained interviewers. Subjects were asked to report the usual frequency and portion size of each food consumed during the past year. The answer for frequency had nine options for each food: "never/almost," "once/month," "2-3 times/month," "1-2 times/week," "3-4 times/week," "5-6 times/week," "once/day," "2 times/day," and " $\geq 3$ times/day." The answer for portion size had three options for each food: "small (1/2 serving/day)," "standard (1 serving/day)," 
and "large ( $\geq 2$ servings / day)." The portion size of each food was determined based on the KoGES FFQ guideline. For analysis, food consumption was converted to weekly frequencies and then multiplied by the reported portion sizes.

To identify dietary patterns, food items were categorized into 27 food groups based on the common food groups classified in the Korean Nutrient Database [16]. Because the grain and grain product intakes of Koreans are very high, this group was divided into seven categories: White rice, whole grains, noodles and dumplings, rice cakes, cereal and snacks, breads, and pizza and hamburger. The vegetable group was divided into two categories: Vegetables and Kimchi (traditional fermented, salted vegetables). Kimchi was considered a single vegetable group because it is frequently eaten as a traditional side dish in Korea and contains high levels of sodium. Also, coffee was separated from the beverage group because the consumption of coffee is common between meals or after meals among Korean adults. Daily nutrient intakes were calculated using a food composition table from the Rural Development Administration of Korea [17].

\subsection{Definition of Low HDL Cholesterolemia and High LDL Cholesterolemia}

Plasma levels of HDL-C and LDL-C were measured every 2 years to calculate incidence of low HDL cholesterolemia or high LDL cholesterolemia during the follow-up period. Low HDL cholesterolemia was defined as a plasma HDL-C level $<40 \mathrm{mg} / \mathrm{dL}(1.04 \mathrm{mmol} / \mathrm{L})$ in men or $<50 \mathrm{mg} / \mathrm{dL}(1.30 \mathrm{mmol} / \mathrm{L})$ in women based on the criteria from the joint interim statement of the International Diabetes Federation and the American Heart Association/National Heart, Lung, and Blood Institute [18]. High LDL cholesterolemia was defined as a plasma LDL-C level $>130 \mathrm{mg} / \mathrm{dL}$ $(3.37 \mathrm{mmol} / \mathrm{L})$ based on the criteria of the National Cholesterol Education Program-Adult Treatment Panel III [19].

\subsection{Other Variables}

Demographic factors, socioeconomic status, and lifestyle factors of the subjects at baseline were determined using questionnaires by trained interviewers. This study collected information on education level ( $\leq 6$ years (elementary school graduate), $7-\leq 12$ years (middle school or high school graduate), and $>12$ years (college graduate or more)), household income level ( $<850$ US $\$$, $850-<1700$ US\$, $1700-<2500$ US\$, and $\geq 2500$ US\$), alcohol intake (never, former, and current), and smoking status (never, former, and current). Physical activity level was assessed using the metabolic equivalent of task (MET) hours per day. Subjects reported hours spent on sleep and activities classified according to activity intensity, including sedentary, very light, light, moderate, and heavy activity [20]. Information on anthropometric and biochemical measurements were collected biennially. Height was measured to the nearest $0.1 \mathrm{~cm}$ using a stadiometer (Aluminum anthropometer, Samhwa Instrument, Seoul, Korea), and body weight was measured to the nearest $0.1 \mathrm{~kg}$ while the subject wore light clothes, without shoes, using bioelectrical impedance analysis (Inbody 3.0, Biospace Corp., Seoul, Korea) by trained research staff. Body mass index (BMI) was calculated as weight (kg) divided by height squared $\left(\mathrm{m}^{2}\right)$. The blood samples were collected after at least eight hours of fasting, and plasma was separated for biochemical testing. The concentrations of TG, total cholesterol, and HDL-C in plasma were measured using an autoanalyzer (ADIVA 1650, Bayer HealthCare, Tarrytown, NY, USA). The concentrations of LDL-C were calculated by the Friedewald equation (total cholesterol-HDL-C-TG/5) [21].

\subsection{Statistical Analyses}

Dietary patterns were separately derived for men and women using factor analysis. Food groups were expressed as the percentage of energy for each food group in order to adjust for weight differences between solid and liquid foods. Food groups were entered into the factor analysis using the FACTOR procedure of SAS software (version 9.4, SAS Institute, Inc., Cary, NC, USA). To identify the number of factors to be retained, we used an eigenvalue $>1.3$ criterion [22]. The factors were rotated with 
an orthogonal transformation using a varimax rotation to achieve a simpler structure with greater interpretability [23]. Post-rotated factor loadings revealed that the four factors well described the distinctive dietary patterns in the study population. Factor scores were saved from the principal component analysis for each individual after varimax rotation. Factor scores for each pattern and for each individual were determined by summing the intake of all food group weighted by the factor loading. Factor scores of each dietary pattern were categorized into tertiles for comparison of lifestyle factors and nutrient intake.

Characteristics of subjects at baseline are expressed as number and percentage (categorical variables) or as mean and standard deviation (continuous variables). Comparison of variables at baseline across tertile categories of dietary pattern scores were performed by the Chi-square test and by generalized linear models, as appropriate. Time-dependent Cox proportional hazard models were used to assess hazard ratios (HRs) and 95\% confidence intervals (CIs) for developing low HDL cholesterolemia or high LDL cholesterolemia according to tertile category of dietary pattern score. The proportional hazard assumption was confirmed graphically using log-log plots [24] and statistically based on Schoenfeld's residuals [25] with no major violation of the assumption. In multivariable adjusted models, age was adjusted in model 1. Age, BMI, residential location, education level, household income, smoking status, alcohol intake, and physical activity were adjusted in model 2. All $p$ values $<0.05$ were considered statistically significant.

\section{Results}

\subsection{Dietary Patterns in Men and Women}

Four dietary patterns were identified for men and women using factor analysis (Table 1). The following four patterns were defined based on the food items that highly loaded: Prudent pattern characterized by high intake of potatoes, legumes, vegetables, mushrooms, fish and shellfish, and seaweed; coffee, fat, and sweet pattern with a higher intake of coffee, oil and fat, and sweets; whole grain pattern (men) or white rice and noodle pattern (women); westernized pattern with high factor loading of cereal and snacks, breads, pizza and hamburgers, nuts, fruits, and dairy products.

Table 1. Factor loading matrix for major factors ascertained from dietary patterns of Korean adults.

\begin{tabular}{|c|c|c|c|c|}
\hline Foods or Food Groups & $\begin{array}{l}\text { Prudent Pattern } \\
\text { (Men/Women) }\end{array}$ & $\begin{array}{l}\text { Coffee, Fat, and Sweet } \\
\text { Pattern (Men/Women) }\end{array}$ & $\begin{array}{l}\text { Whole Grain/White Rice and } \\
\text { Noodle Pattern (Men/Women) }\end{array}$ & $\begin{array}{l}\text { Westernized Pattern } \\
\text { (Men/Women) }\end{array}$ \\
\hline White rice & $-0.16 /-0.12$ & $0.07 /-0.08$ & $-0.89 / 0.89$ & $-0.24 /-0.21$ \\
\hline Whole grains & $-0.08 /-0.26$ & $-0.08 / 0.01$ & $0.94 /-0.89$ & $-0.05 /-0.19$ \\
\hline Rice cakes & $0.01 / 0.15$ & $-0.04 /-0.12$ & $0.08 / 0.02$ & $0.29 / 0.19$ \\
\hline Cereals and snacks & $0.08 / 0.02$ & $0.09 / 0.05$ & $-0.04 / 0.16$ & $0.32 / 0.50$ \\
\hline Breads & $-0.13 / 0.11$ & $0.03 / 0.11$ & $-0.07 / 0.20$ & $0.48 / 0.42$ \\
\hline Starch jelly & $0.14 / 0.33$ & $0.02 / 0.02$ & $0.00 / 0.05$ & $0.00 / 0.05$ \\
\hline Sweets & $0.00 /-0.05$ & $0.94 / 0.90$ & $0.02 / 0.09$ & $-0.01 /-0.07$ \\
\hline Nuts & $0.13 / 0.18$ & $0.02 / 0.01$ & $-0.02 /-0.03$ & $0.33 / 0.39$ \\
\hline Legumes & $0.49 / 0.51$ & $-0.01 / 0.00$ & $0.05 /-0.10$ & $-0.10 /-0.05$ \\
\hline Vegetables & $0.66 / 0.61$ & $-0.12 /-0.08$ & $-0.02 /-0.05$ & $-0.01 /-0.13$ \\
\hline Kimchi & $0.23 / 0.19$ & $0.05 / 0.03$ & $0.05 / 0.08$ & $-0.52 /-0.56$ \\
\hline Mushrooms & $0.50 / 0.53$ & $0.05 /-0.02$ & $0.01 /-0.05$ & $0.24 / 0.18$ \\
\hline Seaweeds & $0.50 / 0.42$ & $0.05 /-0.02$ & $0.00 / 0.01$ & $-0.03 /-0.22$ \\
\hline Dairy products & $0.08 / 0.10$ & $-0.11 /-0.06$ & $0.04 /-0.02$ & $0.43 / 0.26$ \\
\hline Soups & $0.22 / 0.37$ & $0.01 /-0.03$ & $-0.16 / 0.07$ & $0.07 / 0.08$ \\
\hline Seasoning & $0.37 / 0.29$ & $0.06 /-0.06$ & $0.13 / 0.02$ & $-0.33 /-0.39$ \\
\hline Oil and fat & $0.00 /-0.02$ & $0.88 / 0.87$ & $-0.01 / 0.09$ & $0.09 / 0.06$ \\
\hline Coffee & $0.00 / 0.05$ & $0.91 / 0.86$ & $-0.02 / 0.09$ & $-0.03 / 0.02$ \\
\hline Carbonated drinks & $0.00 /-0.03$ & $0.03 / 0.06$ & $-0.32 / 0.15$ & $0.23 / 0.00$ \\
\hline Other beverages & $0.13 / 0.14$ & $-0.03 /-0.09$ & $-0.11 / 0.11$ & $0.28 / 0.26$ \\
\hline
\end{tabular}
Food groups with absolute value of factor loading $>|0.3|$ for each dietary pattern are presented in bold. 


\subsection{Baseline Characteristics of Subjects across Tertiles of Dietary Pattern Score}

A follow-up rate of $75 \%$ was achieved, resulting in 8928 person-years accrued in the low HDL cholesterolemia analysis and 13,636 person-years accrued in the high LDL cholesterolemia analysis. The average follow-up period was 40.1 months. During a six-year follow-up period, a total of 1073 subjects ( 602 men and 471 women) developed low HDL cholesterolemia, and 1485 subjects (632 men and 853 women) developed high LDL cholesterolemia.

The characteristics of Korean adults at baseline according to the tertile categories of dietary pattern scores by gender are shown in Tables 2 and 3. Subjects (both men and women) with higher scores for the prudent pattern were more educated, more likely to live in rural areas, and more likely to have higher income compared to those with low scores for prudent pattern. Subjects (both men and women) with high scores for the coffee, fat, and sweet pattern were younger, more likely to live in rural areas, and more likely to have higher income. Subjects with higher scores for the coffee, fat, and sweet pattern were less likely to have never smoked and more likely to have irregular physical activity in men and were less likely to be a never alcohol drinker in women. Other dietary patterns such as whole grain pattern or white rice and noodle pattern, and westernized pattern were not shown because they were not associated with the risk of cholesterolemia.

Table 2. Characteristics of Korean adults in low high-density lipoprotein (HDL) cholesterolemia analysis according to the tertile $(\mathrm{T})$ categories of dietary pattern scores.

\begin{tabular}{|c|c|c|c|c|c|c|}
\hline & \multicolumn{6}{|c|}{ Low HDL Cholesterolemia Analysis $(n=2655)$} \\
\hline & \multicolumn{3}{|c|}{ Prudent Pattern } & \multicolumn{3}{|c|}{ Coffee, Fat, and Sweet Pattern } \\
\hline & T1 & T2 & T3 & T1 & T2 & T3 \\
\hline Men & & & & & & \\
\hline No. of participants & 572 & 572 & 572 & 572 & 572 & 572 \\
\hline Age (year) ${ }^{1}$ & $56.1 \pm 8.8^{a}$ & $54.5 \pm 8.4^{b}$ & $56.1 \pm 8.7^{\mathrm{a}, *}$ & $57.0 \pm 8.7^{\mathrm{a}}$ & $55.0 \pm 8.7^{b}$ & $54.7 \pm 8.5^{\mathrm{b}, * * *}$ \\
\hline \multicolumn{7}{|l|}{ Residential location (\%) ${ }^{2}$} \\
\hline Ansan(urban) & 58.6 & 41.1 & $48.4^{* * *}$ & 64 & 46.5 & $37.6^{* * *}$ \\
\hline Ansung(rural) & 41.4 & 58.9 & 51.6 & 36 & 53.5 & 62.4 \\
\hline \multicolumn{7}{|l|}{ Education level (\%) } \\
\hline$\leq 6$ years & 24.8 & 15.7 & $17.7^{* * *}$ & 24.8 & 15.2 & $18.2^{* *}$ \\
\hline $7-\leq 12$ years & 59.1 & 60.2 & 58 & 56.5 & 61 & 59.8 \\
\hline$>\overline{12}$ years & 16.1 & 24.1 & 24.3 & 18.7 & 23.8 & 22 \\
\hline \multicolumn{7}{|l|}{$\begin{array}{l}\text { Household income } \\
\text { (US\$/month) }(\%)\end{array}$} \\
\hline$<850$ & 32 & 21.9 & $25.0 * * *$ & 33.6 & 22.2 & $23.1^{* * *}$ \\
\hline $850-<1700$ & 27.1 & 22 & 21.2 & 23.1 & 26.2 & 21 \\
\hline $1700-<2500$ & 17.7 & 19.9 & 15.9 & 17.1 & 16.8 & 19.6 \\
\hline$\geq 2500$ & 23.2 & 36.2 & 37.9 & 26.2 & 34.8 & 36.3 \\
\hline \multicolumn{7}{|l|}{ Smoking status (\%) } \\
\hline Never & 26.1 & 23.6 & 25 & 35.8 & 22 & $16.8^{* * *}$ \\
\hline Former & 40.9 & 42.5 & 37.2 & 37.1 & 47.4 & 36.2 \\
\hline Current & 33 & 33.9 & 37.8 & 27.1 & 30.6 & 47 \\
\hline \multicolumn{7}{|l|}{ Alcohol intake (\%) } \\
\hline Never & 16.6 & 13.3 & 12.9 & 15 & 13.3 & $14.5^{*}$ \\
\hline Former & 9.1 & 6.1 & 6.5 & 8.9 & 4.5 & 8.2 \\
\hline Current & 74.3 & 80.6 & 80.6 & 76.1 & 82.2 & 77.3 \\
\hline \multicolumn{7}{|l|}{ Physical activity } \\
\hline $\mathrm{MET}^{3}$, hour/day & $25.5 \pm 16.7^{\mathrm{a}}$ & $22.6 \pm 15.2^{b}$ & $25.5 \pm 14.8^{\mathrm{a}, *}$ & $27.1 \pm 16.4^{\mathrm{a}}$ & $24.0 \pm 15.1^{\mathrm{b}}$ & $22.4 \pm 15.0^{b, * * *}$ \\
\hline Body mass index $\left(\mathrm{kg} / \mathrm{m}^{2}\right)$ & $23.5 \pm 2.8$ & $23.8 \pm 3.0$ & $23.7 \pm 2.7$ & $23.4 \pm 2.9^{\mathrm{a}}$ & $23.8 \pm 2.7^{b}$ & $23.8 \pm 2.9^{b, *}$ \\
\hline Triglyceride (mmol/L) & $1.5 \pm 1.6^{\mathrm{a}}$ & $1.4 \pm 0.9^{\mathrm{a}, \mathrm{b}}$ & $1.3 \pm 0.8^{\mathrm{b}, *}$ & $1.4 \pm 1.0$ & $1.5 \pm 1.5$ & $1.4 \pm 0.8$ \\
\hline HDL cholesterol (mmol/L) & $1.3 \pm 0.2$ & $1.3 \pm 0.2$ & $1.3 \pm 0.2$ & $1.3 \pm 0.2^{\mathrm{a}}$ & $1.3 \pm 0.2^{\mathrm{a}, \mathrm{b}}$ & $1.2 \pm 0.2^{\mathrm{b}, *}$ \\
\hline $\mathrm{LDL}^{4}$ cholesterol (mmol/L) & $3.0 \pm 0.8$ & $3.1 \pm 0.8$ & $3.1 \pm 0.8$ & $2.9 \pm 0.8^{a}$ & $3.1 \pm 0.8^{b}$ & $3.2 \pm 0.8^{c, * * *}$ \\
\hline \multicolumn{7}{|l|}{ Women } \\
\hline No. of participants & 313 & 313 & 313 & 313 & 313 & 313 \\
\hline Age (year) ${ }^{1}$ & $56.6 \pm 8.6^{\mathrm{a}}$ & $54.8 \pm 8.4^{b}$ & $53.5 \pm 7.8^{b, * * *}$ & $57.5 \pm 8.3^{\mathrm{a}}$ & $53.0 \pm 7.4^{b}$ & $54.3 \pm 8.7^{b, * * *}$ \\
\hline \multicolumn{7}{|l|}{ Residential location (\%) ${ }^{2}$} \\
\hline Ansan(urban) & 59.1 & 40.6 & $54.6^{* * *}$ & 62 & 47.6 & $44.7^{* * *}$ \\
\hline Ansung(rural) & 40.9 & 59.4 & 45.4 & 38 & 52.4 & 55.3 \\
\hline
\end{tabular}


Table 2. Cont.

\begin{tabular}{|c|c|c|c|c|c|c|}
\hline & \multicolumn{6}{|c|}{ Low HDL Cholesterolemia Analysis $(n=2655)$} \\
\hline & \multicolumn{3}{|c|}{ Prudent Pattern } & \multicolumn{3}{|c|}{ Coffee, Fat, and Sweet Pattern } \\
\hline & T1 & $\mathrm{T} 2$ & T3 & T1 & T2 & T3 \\
\hline \multicolumn{7}{|l|}{ Education level (\%) } \\
\hline$\leq 6$ years & 50.2 & 35.8 & $27.2^{* * *}$ & 50.8 & 28.4 & $33.9^{* * *}$ \\
\hline $7-\leq 12$ years & 45 & 55.3 & 64.2 & 43.8 & 65.5 & 55.3 \\
\hline$>12$ years & 4.8 & 8.9 & 8.6 & 5.4 & 6.1 & 10.8 \\
\hline \multicolumn{7}{|l|}{$\begin{array}{l}\text { Household income } \\
\text { (US\$/month) (\%) }\end{array}$} \\
\hline$<850$ & 46.3 & 31.3 & $26.8^{* * *}$ & 42.2 & 29.7 & $32.6^{* * *}$ \\
\hline $850-<1700$ & 24.3 & 20.1 & 26.2 & 28.1 & 22.4 & 20.1 \\
\hline $1700-<2500$ & 13.1 & 20.5 & 17.6 & 9.9 & 19.8 & 21.4 \\
\hline$\geq 2500$ & 16.3 & 28.1 & 29.4 & 19.8 & 28.1 & 25.9 \\
\hline \multicolumn{7}{|l|}{ Smoking status (\%) } \\
\hline Never & 97.1 & 98.1 & 97.8 & 98.4 & 98.1 & 96.5 \\
\hline Former & 1 & 1 & 0.6 & 0.6 & 0.3 & 1.6 \\
\hline Current & 1.9 & 0.9 & 1.6 & 1 & 1.6 & 1.9 \\
\hline \multicolumn{7}{|l|}{ Alcohol intake (\%) } \\
\hline Never & 68.7 & 61 & 64.9 & 76.4 & 58.5 & $59.7^{* * *}$ \\
\hline Former & 1.6 & 2.3 & 0.3 & 1.3 & 1.9 & 1 \\
\hline Current & 29.7 & 36.7 & 34.8 & 22.3 & 39.6 & 39.3 \\
\hline \multicolumn{7}{|l|}{ Physical activity } \\
\hline $\mathrm{MET}^{3}$, hour/day & $22.9 \pm 15.5$ & $23.2 \pm 14.3$ & $22.9 \pm 15.8$ & $24.8 \pm 16.7^{\mathrm{a}}$ & $21.0 \pm 13.9^{b}$ & $23.1 \pm 14.7^{\mathrm{a}, \mathrm{b}, *}$ \\
\hline Body mass index $\left(\mathrm{kg} / \mathrm{m}^{2}\right)$ & $24.3 \pm 3.3$ & $24.5 \pm 3.3$ & $23.9 \pm 3.3$ & $23.9 \pm 3.5$ & $24.6 \pm 3.4$ & $24.1 \pm 3.0$ \\
\hline Triglyceride $(\mathrm{mmol} / \mathrm{L})$ & $1.1 \pm 0.5$ & $1.1 \pm 0.5$ & $1.0 \pm 0.5$ & $1.2 \pm 0.6^{\mathrm{a}}$ & $1.1 \pm 0.4^{\mathrm{a}, \mathrm{b}}$ & $1.0 \pm 0.4^{\mathrm{b}, *}$ \\
\hline HDL cholesterol $(\mathrm{mmol} / \mathrm{L})$ & $1.5 \pm 0.2$ & $1.5 \pm 0.2$ & $1.5 \pm 0.2$ & $1.5 \pm 0.2$ & $1.5 \pm 0.2$ & $1.5 \pm 0.2$ \\
\hline $\mathrm{LDL}^{4}$ cholesterol $(\mathrm{mmol} / \mathrm{L})$ & $3.2 \pm 0.8$ & $3.3 \pm 0.8$ & $3.3 \pm 0.7$ & $3.3 \pm 0.8$ & $3.3 \pm 0.7$ & $3.3 \pm 0.8$ \\
\hline
\end{tabular}

Table 3. Characteristics of Korean adults in high low-density lipoprotein (LDL) cholesterolemia analysis according to the tertile $(\mathrm{T})$ categories of dietary pattern scores.

\begin{tabular}{|c|c|c|c|c|c|c|}
\hline & \multicolumn{6}{|c|}{ High LDL Cholesterolemia Analysis $(n=4005)$} \\
\hline & \multicolumn{3}{|c|}{ Prudent Pattern } & \multicolumn{3}{|c|}{ Coffee, Fat, and Sweet Pattern } \\
\hline & T1 & T2 & T3 & T1 & T2 & T3 \\
\hline Men & & & & & & \\
\hline No. of participants & 685 & 686 & 686 & 685 & 686 & 686 \\
\hline Age (year) ${ }^{1}$ & $56.6 \pm 8.9$ & $55.5 \pm 8.8$ & $55.9 \pm 8.5$ & $57.3 \pm 8.9^{\mathrm{a}}$ & $55.3 \pm 8.5^{\mathrm{b}}$ & $55.4 \pm 8.7^{\mathrm{b}, * * *}$ \\
\hline \multicolumn{7}{|l|}{ Residential location $(\%)^{2}$} \\
\hline Ansan(urban) & 69.2 & 50 & $52.8^{* * *}$ & 72.1 & 55.5 & $44.3^{* * *}$ \\
\hline Ansung(rural) & 30.8 & 50 & 47.2 & 27.9 & 44.5 & 55.7 \\
\hline \multicolumn{7}{|l|}{ Education level (\%) } \\
\hline$\leq 6$ years & 27.9 & 19.2 & $16.6^{* * *}$ & 26.9 & 15.7 & $21.1 * * *$ \\
\hline $7-\leq 12$ years & 57.4 & 60.5 & 60.1 & 56.9 & 61.8 & 59.2 \\
\hline$>12$ years & 14.7 & 20.3 & 23.3 & 16.2 & 22.5 & 19.7 \\
\hline \multicolumn{7}{|c|}{ Household income (US\$/month) (\%) } \\
\hline$<850$ & 33.4 & 24.8 & $24.6^{* * *}$ & 34.6 & 22 & $26.2 * * *$ \\
\hline $850-<1700$ & 30.1 & 24.3 & 24.1 & 27.5 & 28 & 23 \\
\hline $1700-<2500$ & 16.2 & 17.8 & 15.7 & 15.9 & 15.7 & 18.1 \\
\hline$\geq 2500$ & 20.3 & 33.1 & 35.6 & 22 & 34.3 & 32.7 \\
\hline \multicolumn{7}{|l|}{ Smoking status (\%) } \\
\hline Never & 24.8 & 26.7 & 24.1 & 34.6 & 24.5 & $16.5^{* * *}$ \\
\hline Former & 38.4 & 35.6 & 35.7 & 34.6 & 40.7 & 34.4 \\
\hline Current & 36.8 & 37.8 & 40.2 & 30.8 & 34.8 & 49.1 \\
\hline \multicolumn{7}{|l|}{ Alcohol intake (\%) } \\
\hline Never & 19.1 & 19.1 & $16.5 *$ & 19.4 & 16.3 & $19.0 *$ \\
\hline Former & 10.4 & 5.5 & 8.9 & 10.5 & 7.2 & 7.1 \\
\hline Current & 70.5 & 75.4 & 74.6 & 70.1 & 76.5 & 73.9 \\
\hline \multicolumn{7}{|l|}{ Physical activity } \\
\hline $\mathrm{MET}^{3}$, hour/day & $26.5 \pm 16.7^{a}$ & $24.2 \pm 16.0^{\mathrm{b}}$ & $25.6 \pm 15.9^{a, b, *}$ & $27.6 \pm 17.1^{\mathrm{a}}$ & $25.1 \pm 15.5^{b}$ & $23.6 \pm 15.7^{b, * * *}$ \\
\hline Body mass index $\left(\mathrm{kg} / \mathrm{m}^{2}\right)$ & $24.0 \pm 3.0$ & $24.2 \pm 3.0$ & $24.1 \pm 2.9$ & $23.8 \pm 3.0$ & $24.4 \pm 2.9$ & $24.1 \pm 3.0$ \\
\hline Triglyceride (mmol/L) & $1.9 \pm 1.9$ & $1.9 \pm 1.9$ & $1.7 \pm 1.3 *$ & $1.9 \pm 1.5$ & $2.0 \pm 2.0$ & $1.8 \pm 1.6$ \\
\hline
\end{tabular}


Table 3. Cont.

\begin{tabular}{|c|c|c|c|c|c|c|}
\hline & \multicolumn{6}{|c|}{ High LDL Cholesterolemia Analysis $(n=4005)$} \\
\hline & \multicolumn{3}{|c|}{ Prudent Pattern } & \multicolumn{3}{|c|}{ Coffee, Fat, and Sweet Pattern } \\
\hline & T1 & T2 & T3 & T1 & T2 & T3 \\
\hline HDL $^{4}$ cholesterol (mmol/L) & $1.1 \pm 0.3^{\mathrm{a}}$ & $1.1 \pm 0.3^{\mathrm{a}, \mathrm{b}}$ & $1.1 \pm 0.3^{\mathrm{b}, *}$ & $1.1 \pm 0.3$ & $1.1 \pm 0.3$ & $1.1 \pm 0.3$ \\
\hline LDL cholesterol (mmol/L) & $2.5 \pm 0.7$ & $2.5 \pm 0.7$ & $2.6 \pm 0.6$ & $2.5 \pm 0.7$ & $2.5 \pm 0.7$ & $2.6 \pm 0.6$ \\
\hline Women & & & & & & \\
\hline No. of participants & 649 & 650 & 649 & 649 & 650 & 649 \\
\hline Age (year) ${ }^{1}$ & $57.9 \pm 9.2^{\mathrm{a}}$ & $55.5 \pm 9.0^{\mathrm{b}}$ & $55.4 \pm 8.7^{b, * * *}$ & $58.1 \pm 8.7^{\mathrm{a}}$ & $54.7 \pm 8.8^{b}$ & $56.2 \pm 9.4^{c, * * *}$ \\
\hline \multicolumn{7}{|l|}{ Residential location (\%) ${ }^{2}$} \\
\hline Ansan(urban) & 71 & 49.9 & $65.2 * * *$ & 70.1 & 59.5 & $59.5 * * *$ \\
\hline Ansung(rural) & 29 & 50.1 & 34.8 & 29.9 & 40.5 & 40.5 \\
\hline \multicolumn{7}{|l|}{ Education level (\%) } \\
\hline$\leq 6$ years & 57.8 & 41.1 & $39.2 * * *$ & 53.6 & 39.2 & $45.1 * * *$ \\
\hline $7-\leq 12$ years & 39.4 & 50.6 & 53.9 & 43.8 & 53.2 & 47 \\
\hline$>12$ years & 2.8 & 8.3 & 6.9 & 2.6 & 7.6 & 7.9 \\
\hline \multicolumn{7}{|c|}{ Household income (US\$/month) (\%) } \\
\hline$<850$ & 53.3 & 38.5 & $41.3^{* * *}$ & 50.8 & 38 & $44.2 * * *$ \\
\hline $850-<1700$ & 23.4 & 20.3 & 24.4 & 24.2 & 25.2 & 18.6 \\
\hline $1700-<2500$ & 11.6 & 19.4 & 14.9 & 12.5 & 16.5 & 17 \\
\hline$\geq 2500$ & 11.7 & 21.8 & 19.4 & 12.5 & 20.3 & 20.2 \\
\hline \multicolumn{7}{|l|}{ Smoking status (\%) } \\
\hline Never & 97.5 & 97.4 & 96.9 & 98.1 & 97.7 & 96 \\
\hline Former & 0.8 & 0.1 & 0.3 & 0.2 & 0.2 & 0.9 \\
\hline Current & 1.7 & 2.5 & 2.8 & 1.7 & 2.1 & 3.1 \\
\hline \multicolumn{7}{|l|}{ Alcohol intake (\%) } \\
\hline Never & 73.7 & 68 & 72.9 & 82.4 & 68.2 & $63.9^{* * *}$ \\
\hline Former & 1.7 & 1.9 & 1.7 & 1.2 & 2 & 2 \\
\hline Current & 24.6 & 30.1 & 25.4 & 16.3 & 29.8 & 34.1 \\
\hline \multicolumn{7}{|l|}{ Physical activity } \\
\hline $\mathrm{MET}^{3}$, hour/day & $24.0 \pm 16.2$ & $23.0 \pm 14.8$ & $23.3 \pm 16.0$ & $24.3 \pm 16.2$ & $23.3 \pm 15.2$ & $22.8 \pm 15.7$ \\
\hline Body mass index $\left(\mathrm{kg} / \mathrm{m}^{2}\right)$ & $24.6 \pm 3.3$ & $24.5 \pm 3.1$ & $24.6 \pm 3.3$ & $24.2 \pm 3.3^{\mathrm{a}}$ & $25.0 \pm 3.2^{b}$ & $24.6 \pm 3.1^{\mathrm{a}, * * *}$ \\
\hline Triglyceride (mmol/L) & $1.5 \pm 1.0$ & $1.5 \pm 1.0$ & $1.5 \pm 1.0$ & $1.6 \pm 1.2^{\mathrm{a}}$ & $1.5 \pm 0.9^{\mathrm{a}, \mathrm{b}}$ & $1.4 \pm 0.9^{\mathrm{b}, *}$ \\
\hline $\mathrm{HDL}^{4}$ cholesterol $(\mathrm{mmol} / \mathrm{L})$ & $1.1 \pm 0.2^{\mathrm{a}}$ & $1.1 \pm 0.3^{\mathrm{a}, \mathrm{b}}$ & $1.2 \pm 0.3^{\mathrm{b}, *}$ & $1.1 \pm 0.2^{\mathrm{a}}$ & $1.2 \pm 0.3^{b}$ & $1.2 \pm 0.3^{b, * *}$ \\
\hline LDL cholesterol $(\mathrm{mmol} / \mathrm{L})$ & $2.7 \pm 0.5$ & $2.7 \pm 0.5$ & $2.7 \pm 0.5$ & $2.7 \pm 0.5$ & $2.7 \pm 0.4$ & $2.7 \pm 0.5 *$ \\
\hline
\end{tabular}

\subsection{Nutrient and Food Intakes across Tertiles of Dietary Pattern Score}

Nutrient and food intakes for Korean adults according to the tertile categories of dietary pattern scores by gender are shown in Tables 4 and 5 . In both men and women, subjects with high scores for the prudent pattern have higher intakes of energy and other nutrients, except energy from carbohydrates and food groups such as vegetables, meat and dairy. Subjects with high scores for the coffee, fat, and sweet pattern have lower intakes of energy from protein and carotene regardless of gender. 
Table 4. Nutrient and food intakes of Korean in low high-density lipoprotein cholesterolemia analysis according to the tertile (T) categories of dietary pattern scores $(n=2655)^{1}$.

\begin{tabular}{|c|c|c|c|c|c|c|}
\hline & \multicolumn{3}{|c|}{ Prudent Pattern } & \multicolumn{3}{|c|}{ Coffee, Fat, and Sweet Pattern } \\
\hline & T1 & T2 & T3 & T1 & T2 & T3 \\
\hline \multicolumn{7}{|l|}{ Men } \\
\hline No. of participants (1716) & 572 & 572 & 572 & 572 & 572 & 572 \\
\hline Energy (kcal/day) & $1901.4 \pm 558.2^{\mathrm{a}}$ & $2081.2 \pm 571.8^{\mathrm{b}}$ & $2146.2 \pm 653.9^{b, * * *}$ & $2031.0 \pm 656.3^{\mathrm{a}}$ & $2172.9 \pm 563.7^{b}$ & $1924.9 \pm 564.7^{c, * * *}$ \\
\hline \multicolumn{7}{|l|}{ Percentage from energy } \\
\hline Carbohydrate $(\%)$ & $76.2 \pm 5.6^{\mathrm{a}}$ & $72.0 \pm 6.0^{\mathrm{b}}$ & $68.3 \pm 6.6^{c, * * *}$ & $72.4 \pm 8.0$ & $71.5 \pm 6.2$ & $72.5 \pm 6.2 *$ \\
\hline Protein $(\%)$ & $11.7 \pm 1.4^{\mathrm{a}}$ & $13.1 \pm 1.6^{\mathrm{b}}$ & $14.9 \pm 2.2^{c, * * *}$ & $13.5 \pm 2.5^{\mathrm{a}}$ & $13.4 \pm 1.9^{\mathrm{a}}$ & $12.8 \pm 1.9^{b, * * *}$ \\
\hline Fat (\%) & $12.2 \pm 4.5^{\mathrm{a}}$ & $14.8 \pm 4.8^{\mathrm{b}}$ & $16.9 \pm 5.0^{c, * * *}$ & $14.1 \pm 5.9^{\mathrm{a}}$ & $15.1 \pm 4.7^{b}$ & $14.8 \pm 4.7^{\mathrm{a}, \mathrm{b}, *}$ \\
\hline Fiber ( $\mathrm{g} /$ dav $)$ & $4.5 \pm 1.9^{\mathrm{a}}$ & $6.0 \pm 2.1^{b}$ & $7.3 \pm 3.2^{c, * * *}$ & $6.0 \pm 3.2^{\mathrm{a}}$ & $6.2 \pm 2.5^{\mathrm{a}}$ & $5.5 \pm 2.3^{b, * * *}$ \\
\hline Vitamin A ( $\mu \mathrm{g} \mathrm{RE}^{2} /$ day $)$ & $309.3 \pm 193.2^{\mathrm{a}}$ & $468.1 \pm 235.3^{b}$ & $668.3 \pm 415.9^{c, * * * *}$ & $504.3 \pm 422.5^{\mathrm{a}}$ & $507.1 \pm 273.5^{\mathrm{a}}$ & $434.2 \pm 271.6^{b, * *}$ \\
\hline Carotene ( $\mu \mathrm{g} /$ day) & $1517.2 \pm 1024.6^{a}$ & $2344.3 \pm 1337.4^{b}$ & $3430.8 \pm 2340.6^{c, * * *}$ & $2556.4 \pm 2346.0^{a}$ & $2533.7 \pm 1515.1^{\mathrm{a}}$ & $2202.2 \pm 1511.4^{\mathrm{b}, *}$ \\
\hline Vitamin C (mg/day) & $65.4 \pm 38.3^{\mathrm{a}}$ & $99.9 \pm 48.4^{\mathrm{b}}$ & $127.7 \pm 67.5^{c, * * *}$ & $97.1 \pm 68.2^{\mathrm{a}, \mathrm{b}}$ & $104.3 \pm 54.2^{\mathrm{a}}$ & $91.7 \pm 51.4^{\mathrm{b}, *}$ \\
\hline Calcium (mg/day) & $303.8 \pm 175.9^{a}$ & $424.7 \pm 191.2^{b}$ & $540.7 \pm 269.0^{c, * * *}$ & $425.2 \pm 286.0^{\mathrm{a}, \mathrm{b}}$ & $450.1 \pm 214.9^{a}$ & $394.0 \pm 196.1^{\mathrm{b}, * * *}$ \\
\hline Sodium (mg/day) & $2187.5 \pm 1171.9^{\text {a }}$ & $2932.5 \pm 1302.3^{b}$ & $3618.1 \pm 1789.5^{c, * * *}$ & $2934.0 \pm 1797.4$ & $2985.9 \pm 1423.4$ & $2818.3 \pm 1423.4$ \\
\hline Cholesterol (mg/day) & $111.9 \pm 90.9^{\mathrm{a}}$ & $161.7 \pm 99.2^{\mathrm{b}}$ & $209.8 \pm 125.5^{c, * * *}$ & $158.7 \pm 123.9^{\mathrm{a}, \mathrm{b}}$ & $175.0 \pm 104.2^{\mathrm{a}}$ & $149.6 \pm 110.0^{b, * *}$ \\
\hline \multicolumn{7}{|l|}{ Food intake (servings/day) } \\
\hline Grains & $4.6 \pm 1.8^{\mathrm{a}}$ & $4.3 \pm 1.5^{b}$ & $4.0 \pm 1.4^{c, * * *}$ & $4.3 \pm 1.6^{\mathrm{a}}$ & $4.6 \pm 1.6^{\mathrm{b}}$ & $4.0 \pm 1.5^{c, * * *}$ \\
\hline Vegetables & $5.0 \pm 2.9^{a}$ & $7.6 \pm 3.4^{b}$ & $10.6 \pm 5.8^{c, * * *}$ & $8.0 \pm 6.0^{\mathrm{a}}$ & $8.0 \pm 4.1^{\mathrm{a}}$ & $7.3 \pm 4.1^{\mathrm{b}, *}$ \\
\hline Fruit & $2.7 \pm 2.5^{\mathrm{a}}$ & $4.0 \pm 3.0^{b}$ & $4.3 \pm 3.1^{b, * * *}$ & $3.4 \pm 2.9^{a}$ & $4.1 \pm 3.2^{b}$ & $3.5 \pm 2.6^{a, * *}$ \\
\hline Meat & $0.3 \pm 0.3^{a}$ & $0.5 \pm 0.5^{b}$ & $0.6 \pm 0.6^{c, * * *}$ & $0.5 \pm 0.6^{\mathrm{a}}$ & $0.5 \pm 0.4^{\mathrm{a}}$ & $0.4 \pm 0.4^{\mathrm{b}, *}$ \\
\hline Dairy & $0.5 \pm 0.8^{\mathrm{a}}$ & $0.7 \pm 0.8^{b}$ & $0.7 \pm 0.9^{\mathrm{b}, * *}$ & $0.7 \pm 1.0^{\mathrm{a}}$ & $0.7 \pm 0.9^{a}$ & $0.5 \pm 0.6^{b, * * *}$ \\
\hline \multicolumn{7}{|l|}{ Women } \\
\hline No. of participants (939) & 313 & 313 & 313 & 313 & 313 & 313 \\
\hline Energy (kcal/day) & $1782.6 \pm 557.1^{\mathrm{a}}$ & $1908.8 \pm 545.8^{\mathrm{a}}$ & $2074.5 \pm 811.3^{b, * * *}$ & $1820.8 \pm 643.1^{\mathrm{a}}$ & $2116.8 \pm 724.4^{\mathrm{b}}$ & $1828.4 \pm 560.4^{\mathrm{a}, * * *}$ \\
\hline \multicolumn{7}{|l|}{ Percentage from energy } \\
\hline Carbohydrate (\%) & $78.5 \pm 4.9^{\mathrm{a}}$ & $73.8 \pm 5.2^{b}$ & $68.6 \pm 7.2^{c, * * *}$ & $75.4 \pm 7.2^{\mathrm{a}}$ & $72.3 \pm 7.0^{\mathrm{b}}$ & $73.1 \pm 6.8^{b, * * * *}$ \\
\hline Protein $(\%)$ & $11.4 \pm 1.4^{\mathrm{a}}$ & $12.9 \pm 1.4^{\mathrm{b}}$ & $15.1 \pm 2.5^{c, * * *}$ & $12.9 \pm 2.5^{\mathrm{a}}$ & $13.6 \pm 2.4^{b}$ & $13.0 \pm 2.2^{a, * *}$ \\
\hline Fat $(\%)$ & $10.2 \pm 4.0^{\mathrm{a}}$ & $13.3 \pm 4.2^{b}$ & $16.3 \pm 5.4^{c, * * *}$ & $11.6 \pm 5.1^{\mathrm{a}}$ & $14.1 \pm 5.1^{b}$ & $14.0 \pm 5.1^{b, * * *}$ \\
\hline Fiber (g/day) & $4.7 \pm 1.9^{\mathrm{a}}$ & $5.7 \pm 2.1^{b}$ & $7.6 \pm 4.0^{c, * * *}$ & $5.8 \pm 3.1^{\mathrm{a}}$ & $6.6 \pm 3.2^{b}$ & $5.6 \pm 2.8^{a, * * *}$ \\
\hline Vitamin A ( $\mu \mathrm{g} \mathrm{RE}^{2} /$ day $)$ & $298.2 \pm 195.9^{\mathrm{a}}$ & $438.3 \pm 230.0^{b}$ & $727.9 \pm 561.0^{c, * * * *}$ & $461.5 \pm 408.8^{\mathrm{a}}$ & $556.8 \pm 467.0^{b}$ & $446.1 \pm 331.5^{\mathrm{a}, *}$ \\
\hline Carotene ( $\mu \mathrm{g} /$ day) & $1491.4 \pm 1059.6^{\mathrm{a}}$ & $2192.2 \pm 1281.3^{b}$ & $3775.0 \pm 3137.7^{c, * * *}$ & $2410.3 \pm 2315.8^{a, b}$ & $2793.7 \pm 2524.8^{a}$ & $2254.7 \pm 1861.0^{\mathrm{b}, *}$ \\
\hline Vitamin C (mg/day) & $77.5 \pm 46.2^{\mathrm{a}}$ & $106.2 \pm 56.1^{b}$ & $144.4 \pm 78.5^{c, * * *}$ & $102.0 \pm 70.5^{\mathrm{a}}$ & $126.0 \pm 74.7^{b}$ & $100.2 \pm 52.3^{\mathrm{a}, * * * *}$ \\
\hline Calcium (mg/day) & $318.1 \pm 193.0^{\mathrm{a}}$ & $432.0 \pm 188.9^{b}$ & $615.5 \pm 335.7^{c, * * *}$ & $419.2 \pm 274.6^{a}$ & $523.6 \pm 316.4^{b}$ & $422.9 \pm 219.5^{\mathrm{a}, * * *}$ \\
\hline Sodium (mg/day) & $1980.0 \pm 1068.0^{\mathrm{a}}$ & $2497.5 \pm 1214.7^{b}$ & $3327.2 \pm 1879.9^{c, * * *}$ & $2429.8 \pm 1489.3^{a}$ & $2828.4 \pm 1608.4^{b}$ & $2546.5 \pm 1479.1^{\mathrm{a}, \mathrm{b}, *}$ \\
\hline Cholesterol (mg/day) & $83.7 \pm 62.5^{\mathrm{a}}$ & $139.4 \pm 98.3^{\mathrm{b}}$ & $217.4 \pm 171.0^{c, * * *}$ & $119.8 \pm 104.1^{\mathrm{a}}$ & $176.0 \pm 159.8^{b}$ & $144.7 \pm 117.9^{a, * * *}$ \\
\hline
\end{tabular}


Table 4. Cont

\begin{tabular}{|c|c|c|c|c|c|c|}
\hline & \multicolumn{3}{|c|}{ Prudent Pattern } & \multicolumn{3}{|c|}{ Coffee, Fat, and Sweet Pattern } \\
\hline & T1 & T2 & T3 & T1 & T2 & T3 \\
\hline \multicolumn{7}{|c|}{ Food intake (servings/day) } \\
\hline Grains & $3.9 \pm 1.5$ & $3.7 \pm 1.3$ & $3.8 \pm 1.7$ & $3.7 \pm 1.4^{\mathrm{a}}$ & $4.1 \pm 1.8^{\mathrm{b}}$ & $3.6 \pm 1.2^{\mathrm{a}, * * *}$ \\
\hline Vegetables & $5.0 \pm 2.6^{\mathrm{a}}$ & $7.1 \pm 3.4^{b}$ & $11.1 \pm 6.8^{c, * * *}$ & $7.5 \pm 5.5^{\mathrm{a}}$ & $8.5 \pm 5.8^{b}$ & $7.2 \pm 4.3^{\mathrm{a}, *}$ \\
\hline Fruit & $4.4 \pm 3.9^{\mathrm{a}}$ & $5.3 \pm 3.6^{b}$ & $5.4 \pm 4.0^{\mathrm{b}, *}$ & $4.5 \pm 3.9^{\mathrm{a}}$ & $5.9 \pm 3.7^{b}$ & $4.9 \pm 3.6^{\mathrm{a}, * *}$ \\
\hline Meat & $0.2 \pm 0.2^{\mathrm{a}}$ & $0.3 \pm 0.2^{b}$ & $0.5 \pm 0.7^{c, * * *}$ & $0.3 \pm 0.4^{\mathrm{a}}$ & $0.4 \pm 0.5^{b}$ & $0.3 \pm 0.4^{\mathrm{a}, \mathrm{b}, *}$ \\
\hline Dairy & $0.7 \pm 0.9^{\mathrm{a}}$ & $0.9 \pm 0.9^{b}$ & $1.0 \pm 1.0^{\mathrm{b}, * * *}$ & $0.8 \pm 0.9^{\mathrm{a}}$ & $1.0 \pm 1.1^{b}$ & $0.7 \pm 0.8^{\mathrm{a}, * * * *}$ \\
\hline
\end{tabular}

${ }^{1}$ Continuous variables are summarized as mean \pm standard deviation and compared using the generalized linear model ${ }^{2}$ RE: retinol equivalent: a,b,c difference of variables among tertile groups were examined by post-hoc test (Tukey's test for multiple comparisons). The unlike superscripts mean significant differences across tertile groups. ${ }^{*} p$ for trend $<0.05 ;{ }^{* *} p$ for trend $<0.001 ; * * *$ for trend $<0.0001$

Table 5. Nutrient and food intakes of Korean in high low-density lipoprotein cholesterolemia analysis according to the tertile $(\mathrm{T})$ categories of dietary pattern scores $(n=4005){ }^{1}$.

\begin{tabular}{|c|c|c|c|c|c|c|}
\hline & \multicolumn{3}{|c|}{ Prudent Pattern } & \multicolumn{3}{|c|}{ Coffee, Fat, and Sweet Pattern } \\
\hline & T1 & T2 & T3 & T1 & T2 & T3 \\
\hline \multicolumn{7}{|l|}{ Men } \\
\hline No. of participants (2057) & 685 & 686 & 686 & 685 & 686 & 686 \\
\hline Energy (kcal/day) & $1835.6 \pm 540.5^{\mathrm{a}}$ & $2003.4 \pm 569.7^{b}$ & $2163.3 \pm 700.4^{c, * * *}$ & $1974.1 \pm 704.0^{\mathrm{a}}$ & $2138.5 \pm 575.3^{b}$ & $1890.0 \pm 550.3^{c, * * *}$ \\
\hline \multicolumn{7}{|l|}{ Percentage from energy } \\
\hline Carbohydrate $(\%)$ & $76.8 \pm 5.7^{\mathrm{a}}$ & $72.9 \pm 6.0^{b}$ & $68.9 \pm 6.6^{c, * * *}$ & $73.5 \pm 7.9^{a}$ & $72.0 \pm 6.5^{b}$ & $73.1 \pm 6.1^{\mathrm{a}, * *}$ \\
\hline Protein (\%) & $11.5 \pm 1.4^{\mathrm{a}}$ & $13.0 \pm 1.6^{\mathrm{b}}$ & $14.8 \pm 2.2^{c, * * *}$ & $13.2 \pm 2.5^{\mathrm{a}}$ & $13.4 \pm 2.1^{\mathrm{a}}$ & $12.7 \pm 2.0^{\mathrm{b}, * * *}$ \\
\hline Fat $(\%)$ & $11.7 \pm 4.7^{\mathrm{a}}$ & $14.1 \pm 4.8^{b}$ & $16.3 \pm 4.9^{\mathrm{c}, * * *}$ & $13.3 \pm 5.9^{\mathrm{a}}$ & $14.6 \pm 4.9^{b}$ & $14.2 \pm 4.6^{\mathrm{b}, * * *}$ \\
\hline Fiber (g/day) & $4.3 \pm 1.9^{a}$ & $5.8 \pm 2.1^{b}$ & $7.4 \pm 3.3^{c, * * *}$ & $5.9 \pm 3.4^{\mathrm{a}}$ & $6.2 \pm 2.6^{a}$ & $5.5 \pm 2.2^{b, * * *}$ \\
\hline Vitamin A ( $\mu \mathrm{g} \mathrm{RE}^{2} /$ day) & $289.7 \pm 189.4^{\mathrm{a}}$ & $453.6 \pm 240.3^{b}$ & $671.4 \pm 429.3^{c, * * *}$ & $482.8 \pm 424.2^{\mathrm{a}}$ & $501.4 \pm 304.6^{b}$ & $430.8 \pm 276.0^{\mathrm{a}, * *}$ \\
\hline Carotene ( $\mu \mathrm{g} /$ day) & $1441.1 \pm 1006.5^{\mathrm{a}}$ & $2297.5 \pm 1380.8^{b}$ & $3435.1 \pm 2420.4^{c, * * *}$ & $2456.0 \pm 2355.9^{a}$ & $2518.6 \pm 1678.6^{a}$ & $2199.6 \pm 1536.9^{\mathrm{b}, *}$ \\
\hline Vitamin C (mg/day) & $61.1 \pm 38.4^{\mathrm{a}}$ & $95.5 \pm 47.3^{b}$ & $130.9 \pm 69.4^{c, * * *}$ & $94.1 \pm 71.7^{\mathrm{a}}$ & $101.9 \pm 57.2^{b}$ & $91.5 \pm 50.0^{\mathrm{a}, *}$ \\
\hline Calcium (mg/day) & $273.4 \pm 157.8^{\mathrm{a}}$ & $403.2 \pm 183.4^{b}$ & $551.4 \pm 289.8^{c, * * * *}$ & $408.1 \pm 302.5^{\mathrm{a}, \mathrm{b}}$ & $435.0 \pm 221.4^{\mathrm{a}}$ & $385.0 \pm 198.9^{\mathrm{b}, * *}$ \\
\hline Sodium (mg/day) & $2080.1 \pm 1203.8^{a}$ & $2867.8 \pm 1310.0^{\mathrm{b}}$ & $3579.2 \pm 1820.2^{c, * * *}$ & $2810.2 \pm 1864.8$ & $2935.1 \pm 1509.6$ & $2782.9 \pm 1355.4$ \\
\hline Cholesterol (mg/day) & $99.5 \pm 85.7^{\mathrm{a}}$ & $147.6 \pm 93.6^{\mathrm{b}}$ & $212.7 \pm 134.4^{\mathrm{c}, * * *}$ & $148.8 \pm 128.6^{\mathrm{a}}$ & $170.6 \pm 113.8^{\mathrm{b}}$ & $140.5 \pm 103.3^{a, * * *}$ \\
\hline \multicolumn{7}{|l|}{ Food intake (servings/day) } \\
\hline Grains & $4.4 \pm 1.6^{\mathrm{a}}$ & $4.2 \pm 1.5^{\mathrm{b}}$ & $4.1 \pm 1.6^{\mathrm{b}, *}$ & $4.3 \pm 1.7^{\mathrm{a}}$ & $4.5 \pm 1.5^{b}$ & $3.9 \pm 1.4^{\mathrm{c}, * * *}$ \\
\hline Vegetables & $4.7 \pm 3.0^{\mathrm{a}}$ & $7.4 \pm 3.6^{b}$ & $10.6 \pm 5.9^{c, * * * *}$ & $7.6 \pm 6.1$ & $7.9 \pm 4.5$ & $7.2 \pm 4.0$ \\
\hline Fruit & $2.5 \pm 2.6^{\mathrm{a}}$ & $3.8 \pm 3.0^{b}$ & $4.3 \pm 3.5^{c, * * *}$ & $3.2 \pm 3.3^{\mathrm{a}}$ & $3.9 \pm 3.3^{b}$ & $3.5 \pm 2.7^{\mathrm{a}, * *}$ \\
\hline Meat & $0.3 \pm 0.3^{a}$ & $0.4 \pm 0.5^{b}$ & $0.6 \pm 0.5^{c, * * *}$ & $0.4 \pm 0.6^{\mathrm{a}}$ & $0.5 \pm 0.5^{b}$ & $0.4 \pm 0.4^{c, * * *}$ \\
\hline Dairy & $0.4 \pm 0.7^{\mathrm{a}}$ & $0.6 \pm 0.8^{b}$ & $0.7 \pm 0.9^{c, * * *}$ & $0.6 \pm 1.0^{\mathrm{a}}$ & $0.7 \pm 0.8^{\mathrm{a}}$ & $0.5 \pm 0.7^{b, * *}$ \\
\hline
\end{tabular}


Table 5. Cont

\begin{tabular}{|c|c|c|c|c|c|c|}
\hline & \multicolumn{3}{|c|}{ Prudent Pattern } & \multicolumn{3}{|c|}{ Coffee, Fat, and Sweet Pattern } \\
\hline & T1 & T2 & T3 & T1 & T2 & T3 \\
\hline \multicolumn{7}{|l|}{ Women } \\
\hline No. of participants (1948) & 649 & 650 & 649 & 649 & 650 & 649 \\
\hline Energy (kcal/day) & $1678.6 \pm 523.8^{a}$ & $1882.3 \pm 544.5^{\mathrm{b}}$ & $1975.7 \pm 760.8^{c, * * *}$ & $1760.1 \pm 636.0^{\mathrm{a}}$ & $2049.8 \pm 713.5^{\mathrm{b}}$ & $1726.5 \pm 467.5^{a, * * *}$ \\
\hline \multicolumn{7}{|l|}{ Percentage from energy } \\
\hline Carbohydrate (\%) & $79.9 \pm 5.2^{\mathrm{a}}$ & $74.9 \pm 5.3^{b}$ & $70.4 \pm 7.4^{\mathrm{c}, * * *}$ & $77.2 \pm 7.2^{\mathrm{a}}$ & $73.4 \pm 7.3^{\mathrm{b}}$ & $74.7 \pm 6.4^{c, * * *}$ \\
\hline Protein $(\%)$ & $11.1 \pm 1.4^{\mathrm{a}}$ & $12.8 \pm 1.5^{b}$ & $14.8 \pm 2.5^{c, * * *}$ & $12.5 \pm 2.3^{\mathrm{a}}$ & $13.5 \pm 2.6^{b}$ & $12.7 \pm 2.1^{\mathrm{a}, * * * *}$ \\
\hline Fat $(\%)$ & $9.0 \pm 4.1^{\mathrm{a}}$ & $12.4 \pm 4.2^{b}$ & $14.8 \pm 5.5^{c, * * *}$ & $10.3 \pm 5.2^{\mathrm{a}}$ & $13.1 \pm 5.3^{b}$ & $12.6 \pm 4.8^{\mathrm{b}, * * *}$ \\
\hline Fiber (g/day) & $4.4 \pm 1.9^{a}$ & $5.7 \pm 2.1^{b}$ & $7.3 \pm 3.6^{c, * * *}$ & $5.3 \pm 2.6^{\mathrm{a}}$ & $6.6 \pm 3.3^{b}$ & $5.5 \pm 2.5^{\mathrm{a}, * * *}$ \\
\hline Vitamin $\mathrm{A}\left(\mu \mathrm{g} \mathrm{RE}^{2} /\right.$ dav $)$ & $261.1 \pm 179.5^{\mathrm{a}}$ & $435.9 \pm 239.0^{b}$ & $684.3 \pm 512.6^{c, * * * *}$ & $390.4 \pm 315.7^{a}$ & $552.9 \pm 461.9^{b}$ & $437.8 \pm 339.9^{a, * * * *}$ \\
\hline Carotene ( $\mu \mathrm{g} /$ day $)$ & $1351.4 \pm 993.3^{\mathrm{a}}$ & $2212.0 \pm 1337.1^{\mathrm{b}}$ & $3572.4 \pm 2869.5^{c, * * *}$ & $2012.9 \pm 1709.2^{\mathrm{a}}$ & $2847.8 \pm 2555.3^{b}$ & $2274.2 \pm 1922.6^{a, * * * *}$ \\
\hline Vitamin C (mg/day) & $71.0 \pm 45.7^{\mathrm{a}}$ & $102.4 \pm 53.4^{\mathrm{b}}$ & $134.8 \pm 76.1^{c, * * *}$ & $90.6 \pm 60.3^{a}$ & $121.9 \pm 76.6^{\mathrm{b}}$ & $95.6 \pm 51.9^{a, * * * *}$ \\
\hline Calcium (mg/day) & $270.2 \pm 157.7^{a}$ & $424.9 \pm 202.3^{b}$ & $572.1 \pm 328.5^{c, * * * *}$ & $371.2 \pm 258.9^{a}$ & $493.1 \pm 308.6^{b}$ & $402.8 \pm 221.5^{\mathrm{a}, * * *}$ \\
\hline Sodium (mg/day) & $1889.6 \pm 1137.2^{\mathrm{a}}$ & $2561.1 \pm 1205.8^{b}$ & $3312.6 \pm 1879.3^{c, * * *}$ & $2269.5 \pm 1353.7^{\mathrm{a}}$ & $2889.8 \pm 1723.7^{\mathrm{b}}$ & $2603.5 \pm 1514.8^{c, * * *}$ \\
\hline Cholesterol (mg/day) & $64.5 \pm 55.2^{\mathrm{a}}$ & $126.5 \pm 79.4^{\mathrm{b}}$ & $199.2 \pm 175.6^{c, * * *}$ & $110.5 \pm 119.8^{\mathrm{a}}$ & $162.4 \pm 156.7^{\mathrm{b}}$ & $117.2 \pm 93.5^{a, * * *}$ \\
\hline \multicolumn{7}{|l|}{ Food intake (servings/day) } \\
\hline Grains & $3.8 \pm 1.3$ & $3.8 \pm 1.3$ & $3.7 \pm 1.5$ & $3.7 \pm 1.3^{\mathrm{a}}$ & $4.1 \pm 1.6^{\mathrm{b}}$ & $3.5 \pm 1.2^{c, * * *}$ \\
\hline Vegetables & $4.6 \pm 2.7^{\mathrm{a}}$ & $7.1 \pm 3.3^{b}$ & $11.0 \pm 7.0^{c, * * *}$ & $6.7 \pm 4.6^{\mathrm{a}}$ & $8.8 \pm 6.5^{b}$ & $7.2 \pm 4.7^{\mathrm{a}, * * *}$ \\
\hline Fruit & $4.1 \pm 3.9^{\mathrm{a}}$ & $5.0 \pm 3.8^{\mathrm{b}}$ & $4.9 \pm 3.7^{b, * * *}$ & $4.3 \pm 4.0^{\mathrm{a}}$ & $5.5 \pm 4.4^{b}$ & $4.2 \pm 2.7^{\mathrm{a}, * * *}$ \\
\hline Meat & $0.1 \pm 0.2^{\mathrm{a}}$ & $0.2 \pm 0.2^{b}$ & $0.4 \pm 0.8^{c, * * *}$ & $0.2 \pm 0.6^{\mathrm{a}}$ & $0.3 \pm 0.5^{b}$ & $0.2 \pm 0.2^{\mathrm{a}, * *}$ \\
\hline Dairy & $0.5 \pm 0.7^{\mathrm{a}}$ & $0.8 \pm 1.0^{b}$ & $0.9 \pm 1.1^{\mathrm{b}, * * *}$ & $0.7 \pm 1.0^{\mathrm{a}}$ & $0.9 \pm 1.0^{b}$ & $0.7 \pm 0.8^{a, * *}$ \\
\hline
\end{tabular}

${ }^{1}$ Continuous variables are summarized as mean \pm SD and compared using the generalized linear model; ${ }^{2}$ RE: retinol equivalent; ${ }^{\mathrm{a}, \mathrm{b}, \mathrm{c}}$ difference of variables among tertile groups were examined by post-hoc test (Tukey's test for multiple comparisons). The unlike superscripts mean significant differences across tertile groups. ${ }^{*} p$ for trend $<0.05$; ${ }^{* *} p$ for trend $<0.001$; $* * * p$ for trend $<0.0001$ 


\subsection{Association of Dietary Pattern with Low HDL Cholesterolemia or High LDL Cholesterolemia}

The hazard ratios (HRs) and 95\% confidence intervals (CIs) for the risk of incident low HDL cholesterolemia and high LDL cholesterolemia according to dietary pattern score by gender are shown in Table 6. Subjects with high scores for the prudent pattern had a lower risk of low HDL cholesterolemia in both gender groups. Men in the highest tertile of the prudent pattern had a $26 \%$ lower risk of developing low HDL cholesterolemia ( $\mathrm{HR}=0.74,95 \% \mathrm{CI}=0.61-0.91, p$ for trend $=0.0036$ ), and women in the highest tertile of the prudent pattern had a $23 \%$ reduced risk of developing low HDL cholesterolemia $(\mathrm{HR}=0.77,95 \% \mathrm{CI}=0.61-0.96, p$ for trend $=0.0174)$ compared with those in the lowest tertile of the prudent pattern after adjustment for age. The trend remained after adjustment for potential confounders of age, BMI, residential location, education level, household income, smoking status, alcohol intake, and physical activity in both men $(\mathrm{HR}=0.76,95 \% \mathrm{CI}=0.62-0.94, p$ for trend $=0.0098)$ and women $(\mathrm{HR}=0.78,95 \% \mathrm{CI}=0.62-0.98, p$ for trend $=0.0324)$. Other dietary patterns were not associated with the risk of incident low HDL cholesterolemia for either men or women. Men in the highest tertile of the coffee, fat, and sweet pattern had a $26 \%$ increased risk of developing high LDL cholesterolemia $(\mathrm{HR}=1.26,95 \% \mathrm{CI}=1.03-1.55, p$ for trend $=0.0254)$ compared with those in the lowest tertile of the coffee, fat, and sweet pattern after adjustment for potential confounding factors. However, there was no association between the coffee, fat, and sweet pattern and high LDL cholesterolemia in women. Other dietary patterns were not associated with the risk of incident high LDL cholesterolemia for either men or women.

Table 6. Hazard ratios (HRs) and 95\% confidence intervals (CIs) for the risk of incident cholesterolemia according to dietary pattern ${ }^{1}$.

\begin{tabular}{|c|c|c|c|c|}
\hline & T1 & T2 & T3 & $p$ for Trend \\
\hline \multicolumn{5}{|c|}{ Low HDL ${ }^{2}$ Cholesterolemia Analysis $(n=2655)$} \\
\hline \multicolumn{5}{|c|}{ Prudent pattern } \\
\hline \multicolumn{5}{|l|}{ Men } \\
\hline No. of participants (No. of cases) & $572(219)$ & $572(208)$ & $572(175)$ & \\
\hline Model 1 & 1.00 & $0.97(0.80-1.17)$ & $0.74(0.61-0.91)$ & 0.0036 \\
\hline Model 2 & 1.00 & $0.99(0.81-1.20)$ & $0.76(0.62-0.94)$ & 0.0098 \\
\hline \multicolumn{5}{|l|}{ Women } \\
\hline No. of participants (No. of cases) & $313(179)$ & $313(145)$ & $313(147)$ & \\
\hline Model 1 & 1.00 & $0.80(0.64-1.00)$ & $0.77(0.61-0.96)$ & 0.0174 \\
\hline Model 2 & 1.00 & $0.80(0.64-1.00)$ & $0.78(0.62-0.98)$ & 0.0324 \\
\hline \multicolumn{5}{|c|}{ Coffee, fat, and sweet pattern } \\
\hline \multicolumn{5}{|l|}{ Men } \\
\hline No. of participants (No. of cases) & $572(189)$ & $572(203)$ & $572(210)$ & \\
\hline Model 1 & 1.00 & $1.09(0.89-1.33)$ & $1.10(0.90-1.34)$ & 0.3507 \\
\hline Model 2 & 1.00 & $1.06(0.87-1.30)$ & $1.02(0.83-1.26)$ & 0.8364 \\
\hline \multicolumn{5}{|l|}{ Women } \\
\hline No. of participants (No. of cases) & $313(160)$ & $313(156)$ & $313(155)$ & \\
\hline Model 1 & 1.00 & $1.06(0.84-1.33)$ & $1.12(0.90-1.40)$ & 0.3197 \\
\hline Model 2 & 1.00 & $1.01(0.80-1.27)$ & $1.15(0.91-1.44)$ & 0.2370 \\
\hline \multicolumn{5}{|c|}{ High LDL ${ }^{3}$ Cholesterolemia Analysis $(n=4005)$} \\
\hline \multicolumn{5}{|c|}{ Prudent pattern } \\
\hline Men & & & & \\
\hline No. of participants (No. of cases) & $685(214)$ & $686(213)$ & $686(205)$ & \\
\hline Model 1 & 1.00 & $1.03(0.85-1.24)$ & $0.97(0.80-1.18)$ & 0.7678 \\
\hline Model 2 & 1.00 & $0.95(0.78-1.15)$ & $0.90(0.74-1.10)$ & 0.3124 \\
\hline
\end{tabular}


Table 6. Cont.

\begin{tabular}{|c|c|c|c|c|}
\hline & T1 & T2 & T3 & $p$ for Trend \\
\hline Women & & & & \\
\hline No. of participants (No. of cases) & $649(285)$ & $650(299)$ & 649 (269) & \\
\hline Model 1 & 1.00 & $1.02(0.87-1.20)$ & $0.90(0.76-1.07)$ & 0.2175 \\
\hline Model 2 & 1.00 & $0.97(0.82-1.15)$ & $0.88(0.75-1.05)$ & 0.1512 \\
\hline \multicolumn{5}{|c|}{ Coffee, fat, and sweet pattern } \\
\hline \multicolumn{5}{|l|}{ Men } \\
\hline No. of participants (No. of cases) & $685(180)$ & $686(210)$ & $686(242)$ & \\
\hline Model 1 & 1.00 & $1.14(0.94-1.40)$ & $1.36(1.12-1.65)$ & 0.0017 \\
\hline Model 2 & 1.00 & $1.08(0.88-1.33)$ & $1.26(1.03-1.55)$ & 0.0254 \\
\hline \multicolumn{5}{|l|}{ Women } \\
\hline No. of participants (No. of cases) & $649(283)$ & $650(284)$ & $649(286)$ & \\
\hline Model 1 & 1.00 & $0.98(0.83-1.16)$ & $0.99(0.84-1.16)$ & 0.8771 \\
\hline Model 2 & 1.00 & $0.97(0.82-1.15)$ & $0.97(0.82-1.14)$ & 0.6913 \\
\hline
\end{tabular}

${ }^{1}$ Calculated using the Cox proportional hazard model; Model 1 adjusted for age; Model 2 adjusted for age, body mass index, residential location, education level, household income, smoking status, alcohol intake and physical activity. ${ }^{2}$ HDL: High-density lipoprotein; ${ }^{3}$ LDL: low-density lipoprotein.

\section{Discussion}

In this large cohort study, specific Korean dietary patterns were associated with the risk of incident dyslipidemia in middle-aged and older Korean adults. Using factor analysis, four dietary patterns were derived from Korean men and women: prudent pattern, coffee, fat, and sweet pattern, whole grain (men) or white rice and noodle (women) pattern, and westernized pattern. During a six-year follow-up period, the prudent pattern characterized by high intakes of potatoes, legumes, vegetables, mushrooms, fish and shellfish, and seaweed was associated with a $22-24 \%$ lower risk of developing low HDL cholesterolemia after adjustment for potential confounders in both men and women. In men only, a coffee, fat, and sweet pattern characterized by high intakes of coffee, oil and fat, and sweets was associated with a $26 \%$ higher risk of developing high LDL cholesterolemia after adjustment for potential confounding factors. Other dietary patterns were not significantly associated with cholesterolemia in either men or women. These results suggest that a specific dietary pattern might be an independent determinant of cholesterolemia with gender difference in the association between diet and cholesterolemia.

Food composition of the prudent pattern that was inversely associated with low HDL cholesterolemia in this study is similar to the Mediterranean-style diet or DASH diet, which is representative of a healthy dietary pattern, as shown in Western populations. In the Framingham Heart Study, in a seven-year follow-up period, US adults with a higher score regarding the Mediterranean-style pattern had a 32\% higher level of HDL-C compared to those with a low pattern score after adjustment for potential confounders [7]. In a cross-sectional study of a Spanish population aged 40-55 years, participants adhering to the Mediterranean dietary pattern had $3.3 \mathrm{mg} / \mathrm{dL}$ higher HDL-C levels [8]. The DASH diet was inversely associated with low HDL-C. In a cross-sectional study of Iranian women aged over 30 years, participants in the highest tertile of DASH diet scores were associated with a 78\% lower risk of low HDL cholesterolemia compared with those in the lowest tertile of DASH diet scores [26]. Similar to the prudent pattern shown in the present study, in a Greek population aged over 18 years, a dietary pattern with high intakes of fish, vegetables, legumes, cereals, and fruits was positively associated with high HDL-C concentration [6].

There are possible mechanisms to explain an inverse association between a prudent dietary pattern and low HDL cholesterolemia. The prudent dietary pattern includes various foods and food groups that are rich in nutrients and food components such as dietary fiber, antioxidants (beta-carotene, vitamins $C$ and $E$ ), polyphenols, dietary fat, and some minerals including calcium, which influence health outcomes. In the present study, participants with higher scores for the prudent pattern had 
higher intakes of fiber, carotene, vitamin $C$, and calcium. In a randomized controlled trial of a Swedish population aged 25-65 years, the diet, which was high in dietary fiber, beta-glucan, and polyunsaturated fat for six weeks, was associated with a 5\% decrease of HDL-C concentration [27]. Vegetables and legumes, major components of the prudent pattern and contributors to dietary fiber intake, are known to have beneficial effects on blood cholesterol levels [28,29]. Frequent legume consumption (>1 time/day) was associated with a 19\% lower risk of low HDL cholesterolemia compared to infrequent legume consumption (<3 times/week) in Iranian adults aged $\geq 19$ years [28]. A meta-analysis of 60 controlled trials reported that replacing carbohydrate with fat such as saturated fatty acids, monounsaturated fatty acids, or polyunsaturated fatty acids in diet increased serum HDL cholesterol [30]. Also, omega-3 fatty acids, which are abundant in fish, shellfish, and seaweed, have beneficial effects on serum HDL-C concentration [31,32]. Omega-3 supplementation (2.4 g/day) for eight weeks led to a significant increase in serum HDL level compared with a placebo group in Iranian adolescents aged 10 to 18 years [31]. Calcium intake was inversely associated with elevated total cholesterol and reduced HDL-C level in urban Korean adults aged over 20 years [33]. Altogether, these findings show that the protective effects of the prudent pattern on low HDL cholesterolemia might be due to the combined and synergistic effects of various components, rather than a single nutrient or food component.

It is of interest that the coffee, fat, and sweet pattern was positively associated with high LDL cholesterolemia in men only. Consistent with the present study, a meta-analysis of randomized trials conducted in Western countries revealed positive dose-response relationships between coffee intake and total cholesterol and LDL-C. On average, drinking coffee for 45 days was associated with an increase of $8.1 \mathrm{mg} / \mathrm{dL}$ for total cholesterol and $5.4 \mathrm{mg} / \mathrm{dL}$ for LDL-C [34]. Coffee oils might be major contributors to the cholesterol-raising effect of coffee. Coffee oils such as cafestol and kahweol may reduce the activity of hepatic LDL receptors and thereby cause extracellular accumulation of LDL-C [35,36]. A previous meta-analysis showed that trials using unfiltered coffee and boiled coffee had a stronger cholesterol-raising effect than did those using filtered coffee because of a higher concentration of coffee oils [34]. The LDL-C raising effect of cafestol might be affected by the apoprotein E polymorphism, and the effect of apoprotein $\mathrm{E}$ on the response of cholesterol level to the changes in dietary cafestol differed between men and women [37]. This might partly explain gender differences in the association between coffee, fat, and sweet pattern and the risk of high LDL cholesterolemia as revealed in the present study.

In agreement with our study, a meta-analysis has reported gender differences in the effect of coffee consumption on lipid levels [34]. The underlying mechanism for gender differences on the relationship between dietary factors and disease risk has not been clearly revealed. Gender difference might be associated with sex hormones, sex-specific gene polymorphism, or increased immune inflammatory factors that are related to the regulation of lipid levels [12,38]. Sex hormones such as estrogen lower LDL-C and lipoprotein (a) levels and raise HDL-C level, whereas androgens have an adverse effect on lipid profiles [39]. Also, there are many different pathophysiological and social reasons why the relationship between diet and dyslipidemia may have been only observed in men. Although the present study was unable to specifically determine the reasons, these results propose that gender should be considered in the analysis of association between diet and dyslipidemia, and more research is needed to determine the underlying mechanism.

Older Korean adults commonly drink instant coffee mix or coffee with sugar (sweets) and powered creamer (oil and fat) between meals or after meals [40]. Powdered creamer is composed of palm oil, sweetener, and some additives. Palm oil is well known to have an adverse effect on health-as much as animal fat [41]. In a meta-analysis of clinical trials, palm oil was positively associated with a $0.24 \mathrm{mmol} / \mathrm{L}$ increase of LDL-C compared with vegetable oils [42]. Thus, fat sources, perhaps the powered creamer, included in the coffee, fat, and sweet pattern as well as coffee oil might also contribute to the positive association between the coffee, fat, and sweet pattern and high LDL 
cholesterolemia. Unfortunately, the fat sources including individual fatty acids were not analyzed in this study.

The strength of this study is that, to the best of our knowledge, it is the first to reveal a causal relationship between dietary pattern and the risk of incident dyslipidemia in the Asian population using data from a large scale cohort study with a long period of follow up ( six years). Also, this is a well-established prospective cohort study that used standardized procedures to collect data on exposure and outcomes. However, this study also has certain limitations. Our study population included only middle-aged and older adults, and the findings might not be generalizable to other age groups. Also, the dietary pattern analysis utilized was somewhat subjective regarding decisions on the number of factors extracted and food groupings. In addition, the random measurement error caused by within-person variation in dietary assessment may exist due to the use of the single time-point of FFQ.

\section{Conclusions}

During a six-year follow up period, a prudent dietary pattern characterized by high intake of potatoes, legumes, vegetables, mushrooms, fish and shellfish, and seaweed was associated with a lower risk of developing low HDL cholesterolemia in both Korean men and women. A coffee, fat, and sweet pattern with high intake of coffee, oil and fat, and sweets was associated with a higher risk of developing high LDL cholesterolemia in men only, suggesting gender differences regarding the association between dietary pattern and the risk of cholesterolemia in middle-aged and older Korean adults. In future studies, randomized clinical trials should be conducted to confirm the association between dietary pattern and risks of dyslipidemia and also to reveal the underlying mechanism of this association.

Acknowledgments: Epidemiologic data used in this study were obtained from the Korean Genome and Epidemiology Study (KoGES; 4851-302), Korea Centers for Disease Control and Prevention, Korea. This research was supported by the Basic Science Research Program of the National Research Foundation of Korea (NRF), funded by the Ministry of Education, Science, and Technology (NRF2016 R1D1A1B03931307). The NRF had no role in the study design, data analysis, or writing of this article.

Author Contributions: Both authors had a significant role in the project. J.L. contributed to data analysis and writing of the manuscript. J.K. contributed to designing the research and writing the manuscript; she also has primary responsibility for the final content. Both authors read and approved the final manuscript.

Conflicts of Interest: The authors declare no conflict of interest.

\section{References}

1. Haffner, S.M. Diabetes, hyperlipidemia, and coronary artery disease. Am. J. Cardiol. 1999, 83, 17F-21F. [CrossRef]

2. Musunuru, K. Atherogenic dyslipidemia: Cardiovascular risk and dietary intervention. Lipids 2010, 45, 907-914. [CrossRef] [PubMed]

3. Toth, P.P.; Potter, D.; Ming, E.E. Prevalence of lipid abnormalities in the United States: The National Health and Nutrition Examination Survey 2003-2006. J. Clin. Lipidol. 2012, 6, 325-330. [CrossRef] [PubMed]

4. Roh, E.; Ko, S.H.; Kwon, H.S.; Kim, N.H.; Kim, J.H.; Kim, C.S.; Song, K.H.; Won, J.C.; Kim, D.J.; Choi, S.H.; et al. Prevalence and management of dyslipidemia in Korea: Korea National Health And Nutrition Examination Survey during 1998 to 2010. Diabetes Metab. J. 2013, 37, 433-449. [CrossRef] [PubMed]

5. Asghari, G.; Yuzbashian, E.; Mirmiran, P.; Hooshmand, F.; Najafi, R.; Azizi, F. Dietary approaches to stop hypertension (DASH) dietary pattern is associated with reduced incidence of metabolic syndrome in children and adolescents. J. Pediatr. 2016, 174, 178-184.e1. [CrossRef] [PubMed]

6. Panagiotakos, D.B.; Pitsavos, C.; Skoumas, Y.; Stefanadis, C. The association between food patterns and the metabolic syndrome using principal components analysis: The ATTICA study. J. Am. Diet. Assoc. 2007, 107, 979-987. [CrossRef] [PubMed] 
7. Rumawas, M.E.; Meigs, J.B.; Dwyer, J.T.; McKeown, N.M.; Jacques, P.F. Mediterranean-style dietary pattern, reduced risk of metabolic syndrome traits, and incidence in the FRAMINGHAM offspring cohort. Am. J. Clin. Nutr. 2009, 90, 1608-1614. [CrossRef] [PubMed]

8. Penalvo, J.L.; Oliva, B.; Sotos-Prieto, M.; Uzhova, I.; Moreno-Franco, B.; Leon-Latre, M.; Ordovas, J.M. Greater adherence to a Mediterranean dietary pattern is associated with improved plasma lipid profile: The Aragon Health Workers Study cohort. Rev. Esp. Cardiol. (Engl. Ed.) 2015, 68, 290-297. [CrossRef] [PubMed]

9. Lyu, S.; Su, J.; Xiang, Q.; Wu, M. Association of dietary pattern and physical activity level with triglyceride to high-density lipoprotein cholesterol ratio among adults in Jiangsu, China: A cross-sectional study with sex-specific differences. Nutr. Res. 2014, 34, 674-681. [CrossRef] [PubMed]

10. Na, L.; Han, T.; Zhang, W.; Wu, X.; Na, G.; Du, S.; Li, Y.; Sun, C. A snack dietary pattern increases the risk of hypercholesterolemia in Northern Chinese adults: A prospective cohort study. PLoS ONE 2015, 10, e0134294. [CrossRef] [PubMed]

11. Chiu, S.; Bergeron, N.; Williams, P.T.; Bray, G.A.; Sutherland, B.; Krauss, R.M. Comparison of the DASH (dietary approaches to stop hypertension) diet and a higher-fat dash diet on blood pressure and lipids and lipoproteins: A randomized controlled trial. Am. J. Clin. Nutr. 2016, 103, 341-347. [CrossRef] [PubMed]

12. Kang, Y.; Kim, J. Gender difference on the association between dietary patterns and metabolic syndrome in Korean population. Eur. J. Nutr. 2016, 55, 2321-2330. [CrossRef] [PubMed]

13. Choi, H.; Song, S.; Kim, J.; Chung, J.; Yoon, J.; Paik, H.Y.; Song, Y. High carbohydrate intake was inversely associated with high-density lipoprotein cholesterol among Korean adults. Nutr. Res. 2012, 32, 100-106. [CrossRef] [PubMed]

14. Kim, D.; Kim, J. Dairy consumption is associated with a lower incidence of the metabolic syndrome in middle-aged and older Korean adults: The Korean Genome and Epidemiology Study (KoGES). Br. J. Nutr. 2017, 117, 148-160. [CrossRef] [PubMed]

15. Ahn, Y.; Kwon, E.; Shim, J.E.; Park, M.K.; Joo, Y.; Kimm, K.; Park, C.; Kim, D.H. Validation and reproducibility of food frequency questionnaire for Korean Genome Epidemiologic Study. Eur. J. Clin. Nutr. 2007, 61, 1435-1441. [CrossRef] [PubMed]

16. Korean Nutrition Society. Dietary Reference Intake for Korean; Kookjin Press: Seoul, Korea, 2010.

17. National Institute of Agricultural Sciences. Food Composition Table, 5th ed.; Rural Development Administration of Korea: Jeonju city, Korea, 1996.

18. Alberti, K.G.; Eckel, R.H.; Grundy, S.M.; Zimmet, P.Z.; Cleeman, J.I.; Donato, K.A.; Fruchart, J.C.; James, W.P.; Loria, C.M.; Smith, S.C., Jr.; et al. Harmonizing the metabolic syndrome: A joint interim statement of the International Diabetes Federation task force on epidemiology and prevention; National Heart, Lung, and Blood Institute; American Heart Association; World Heart Federation; International Atherosclerosis Society; and International Association for the study of Obesity. Circulation 2009, 120, 1640-1645. [PubMed]

19. National Cholesterol Education Program (NCEP) Expert Panel on Detection, Evaluation, and Treatment of High Blood Cholesterol in Adults (Adult Treatment Panel III). Third report of the National Cholesterol Education Program (NCEP) expert panel on detection, evaluation, and treatment of high blood cholesterol in adults (Adult Treatment Panel III) final report. Circulation 2002, 106, 3143-3421.

20. Ainsworth, B.E.; Haskell, W.L.; Whitt, M.C.; Irwin, M.L.; Swartz, A.M.; Strath, S.J.; O’Brien, W.L.; Bassett, D.R., Jr.; Schmitz, K.H.; Emplaincourt, P.O.; et al. Compendium of physical activities: An update of activity codes and met intensities. Med. Sci. Sports Exerc. 2000, 32, S498-S504. [CrossRef] [PubMed]

21. Friedewald, W.T.; Levy, R.I.; Fredrickson, D.S. Estimation of the concentration of low-density lipoprotein cholesterol in plasma, without use of the preparative ultracentrifuge. Clin. Chem. 1972, 18, 499-502. [PubMed]

22. Kim, J.; Jo, I. Grains, vegetables, and fish dietary pattern is inversely associated with the risk of metabolic syndrome in South Korean adults. J. Am. Diet. Assoc. 2011, 111, 1141-1149. [CrossRef] [PubMed]

23. McNaughton, S.A.; Ball, K.; Mishra, G.D.; Crawford, D.A. Dietary patterns of adolescents and risk of obesity and hypertension. J. Nutr. 2008, 138, 364-370. [PubMed]

24. Hess, K.R. Graphical methods for assessing violations of the proportional hazards assumption in Cox regression. Stat. Med. 1995, 14, 1707-1723. [CrossRef] [PubMed]

25. Schoenfeld, D. Partial residuals for the proportional hazards regression model. Biome 1982, 69, $239-241$. [CrossRef] 
26. Saneei, P.; Fallahi, E.; Barak, F.; Ghasemifard, N.; Keshteli, A.H.; Yazdannik, A.R.; Esmaillzadeh, A. Adherence to the DASH diet and prevalence of the metabolic syndrome among Iranian women. Eur. J. Nutr. 2015, 54, 421-428. [CrossRef] [PubMed]

27. Adamsson, V.; Reumark, A.; Fredriksson, I.B.; Hammarstrom, E.; Vessby, B.; Johansson, G.; Riserus, U. Effects of a healthy Nordic diet on cardiovascular risk factors in hypercholesterolaemic subjects: A randomized controlled trial (NORDIET). J. Intern. Med. 2011, 269, 150-159. [CrossRef] [PubMed]

28. Mohammadifard, N.; Sarrafzadegan, N.; Paknahad, Z.; Nouri, F. Inverse association of legume consumption and dyslipidemia: Isfahan healthy heart program. J. Clin. Lipidol. 2014, 8, 584-593. [CrossRef] [PubMed]

29. Hosseinpour-Niazi, S.; Mirmiran, P.; Amiri, Z.; Hosseini-Esfahani, F.; Shakeri, N.; Azizi, F. Legume intake is inversely associated with metabolic syndrome in adults. Arch. Iran. Med. 2012, 15, 538-544. [PubMed]

30. Mensink, R.P.; Zock, P.L.; Kester, A.D.; Katan, M.B. Effects of dietary fatty acids and carbohydrates on the ratio of serum total to HDL cholesterol and on serum lipids and apolipoproteins: A meta-analysis of 60 controlled trials. Am. J. Clin. Nutr. 2003, 77, 1146-1155. [PubMed]

31. Ahmadi, A.; Gharipour, M.; Arabzadeh, G.; Moin, P.; Hashemipour, M.; Kelishadi, R. The effects of vitamin E and omega-3 pufas on endothelial function among adolescents with metabolic syndrome. Biomed. Res. Int. 2014, 2014, 906019. [CrossRef] [PubMed]

32. Ferguson, J.J.; Veysey, M.; Lucock, M.; Niblett, S.; King, K.; MacDonald-Wicks, L.; Garg, M.L. Association between omega-3 index and blood lipids in older Australians. J. Nutr. Biochem. 2016, 27, 233-240. [CrossRef] [PubMed]

33. Song, S.; Paik, H.Y.; Park, M.; Song, Y. Dyslipidemia patterns are differentially associated with dietary factors. Clin. Nutr. 2016, 35, 885-891. [CrossRef] [PubMed]

34. Cai, L.; Ma, D.; Zhang, Y.; Liu, Z.; Wang, P. The effect of coffee consumption on serum lipids: A meta-analysis of randomized controlled trials. Eur. J. Clin. Nutr. 2012, 66, 872-877. [CrossRef] [PubMed]

35. Ranheim, T.; Halvorsen, B. Coffee consumption and human health-Beneficial or detrimental?-Mechanisms for effects of coffee consumption on different risk factors for cardiovascular disease and type 2 diabetes mellitus. Mol. Nutr. Food Res. 2005, 49, 274-284. [CrossRef] [PubMed]

36. Rustan, A.C.; Halvorsen, B.; Huggett, A.C.; Ranheim, T.; Drevon, C.A. Effect of coffee lipids (cafestol and kahweol) on regulation of cholesterol metabolism in hepg2 cells. Arterioscler. Thromb. Vasc. Biol. 1997, 17, 2140-2149. [CrossRef] [PubMed]

37. Weggemans, R.M.; Zock, P.L.; Ordovas, J.M.; Pedro-Botet, J.; Katan, M.B. Apoprotein E genotype and the response of serum cholesterol to dietary fat, cholesterol and cafestol. Atherosclerosis 2001, 154, 547-555. [CrossRef]

38. Mosher, M.J.; Martin, L.J.; Cupples, L.A.; Yang, Q.; Dyer, T.D.; Williams, J.T.; North, K.E. Genotype-by-sex interaction in the regulation of high-density lipoprotein: The Framingham Heart Study. Hum. Biol. 2005, 77, 773-793. [CrossRef] [PubMed]

39. Rossouw, J.E. Hormones, genetic factors, and gender differences in cardiovascular disease. Cardiovasc. Res. 2002, 53, 550-557. [CrossRef]

40. Je, Y.; Jeong, S.; Park, T. Coffee consumption patterns in Korean adults: The Korean National Health and Nutrition Examination Survey (2001-2011). Asia Pac. J. Clin. Nutr. 2014, 23, 691-702. [PubMed]

41. Kim, H.J.; Cho, S.; Jacobs, D.R., Jr.; Park, K. Instant coffee consumption may be associated with higher risk of metabolic syndrome in Korean adults. Diabetes Res. Clin. Pract. 2014, 106, 145-153. [CrossRef] [PubMed]

42. Sun, Y.; Neelakantan, N.; Wu, Y.; Lote-Oke, R.; Pan, A.; van Dam, R.M. Palm oil consumption increases LDL cholesterol compared with vegetable oils low in saturated fat in a meta-analysis of clinical trials. J. Nutr. 2015, 145, 1549-1558. [CrossRef] [PubMed]

(C) 2018 by the authors. Licensee MDPI, Basel, Switzerland. This article is an open access article distributed under the terms and conditions of the Creative Commons Attribution (CC BY) license (http:/ / creativecommons.org/licenses/by/4.0/). 